\title{
A-02
}

\section{AUTOMATIZACIÓN DEL RIEGO POR SUPERFICIE ¿UNA UTOPÍA?}

\author{
Monserrat, J. ${ }^{1}$, Cots, L. ${ }^{2}$, Lordán, I. ${ }^{3}$ Llorens J. ${ }^{4}$, Ferré, P. ${ }^{5}$ \\ 1,2,4 Profesor. Dep. Ingenieria Agroforestal, Universidad de Lleida, monserrat@eagrof.udl.cat \\ 3,5 Becario, Dep. Ingenieria Agroforestal, Universidad de Lleida.
}

\section{Resumen}

La automatización del riego por superficie puede ser una alternativa a la modernización a riego a presión. Se presentan los resultados de unos ensayos de compuertas automáticas para acequias y parcelas que incorporan un control telemático de las mismas. Las compuertas han realizado su función correctamente. Las evaluaciones del riego realizadas muestran que el agricultor tiene una tendencia a aplicar dosis mayores que las óptimas, pensamos que la automatización puede ser un medio para reducir dichas dosis. También se presenta una expresión sencilla para calcular el precio máximo de la automatización por debajo del cual es rentable la automatización.

\section{Introducción}

El riego por superficie es el método de riego más antiguo, no obstante ha ido incorporando mejoras a lo largo de su historia, como son el riego con acequias de hormigón y tajaderas que ahorra el trabajo con la azada. Otra mejora introducida ha sido la nivelación láser que permite una distribución más uniforme del agua.

Cuando se plantea una modernización del riego por superficie se suele pensar en la transformación a presión, sin embargo actualmente existen compuertas automáticas que incorporan tecnologías de la información al riego por superficie que pueden situarlo como una alternativa a considerar en la modernización.

El riego por superficie presenta la ventaja de no necesitar agua a presión y por tanto su nulo coste energético. Además su modernización requiere una inversión inferior.

Lógicamente también tiene limitaciones como son un menor control de la dosis aplicada, la cual es alta, por lo que necesita suelos con alta capacidad de retención. Al aplicar dosis altas los intervalos de riego son elevados lo cual también tiene una repercusión negativa en la producción. Finalmente cabría añadir la necesidad de mano de obra para su operación. Justamente en este aspecto es donde principalmente incide los nuevos desarrollos en automatización.

Respecto al aprovechamiento del agua en el riego por superficie, estudios realizados por la Universidad de Lleida (Cots, Clop, \& Lopez, 2007) comprueban que si se cumplen ciertas condiciones como son terrenos llanos, con suelos profundos y con alta retención de agua la eficiencia del uso del agua es equiparable a la de otros sistemas de riego.

En el riego por superficie se pueden distinguir dos grados de automatización: La semiautomatización en que solo se automatiza alguna de las fases del riego (p.e. abrir una compuerta que conduzca el agua al siguiente tablar); y la automatización completa en que todas las fases están automatizadas. Las compuertas semiautomáticas tienen la ventaja de que liberan al agricultor de estar pendiente del riego y por otra parte son más económicas. 
El grupo de riegos de la universidad de Lleida ha desarrollado prototipos de compuertas semiautomáticas (Monserrat, Rosell, Barragán, Cots, \& Pastor, 1998) y actualmente está desarrollando otra con un módulo de telecomunicación con el usuario que le informa de las acciones que ha realizado y si ha habido algún problema.

\section{Objetivos}

En la comunicación se presentan los resultados de unos ensayos realizados con equipos comerciales de compuertas automáticas en una comunidad de regantes de los canales de Urgel.

Otro aspecto a tratar en la comunicación es la propuesta de un indicador económico sencillo que permita evaluar el precio máximo de inversión a partir del cual la automatización no es competitiva con los precios actuales de la mano de obra para el riego.

\section{Material y Métodos}

El ensayo se ha realizado la campaña 2016 en la acequia de la Serreta del Canal de Urgell en el término municipal de Arbeca. En la parte media de dicha acequia había una balsa reguladora con el fin de no tener que regar de noche.

En la salida de la balsa se ha instalado una compuerta automática de tajadera, el modelo Slipmeter ( Fig. 1), marca Rubicon Water, con la función de abrir y cerrar. También tiene capacidad de medir el caudal y el volumen servido y regular a partir de estas variables. Está compuesta de un accionador, un módulo de operación y otro de comunicaciones, todo alimentado por una placa solar, además a la entrada dispone de un caudalímetro electromagnético multihaz (Fig. 2). En dicha compuerta se programó un horario de apertura y cierre y un caudal de consigna. El sistema permite disponer de información en tiempo real de su funcionamiento y se puede modificar los parámetros a distancia, mediante una aplicación móvil.

Aguas abajo de la compuerta se ha construido un aforador para registrar la evolución del caudal con el tiempo y contrastarlo con el que medía la compuerta automática.

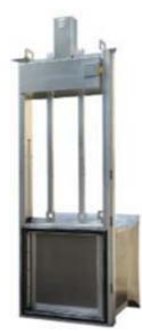

Figura 1. Compuerta de tajadera automática

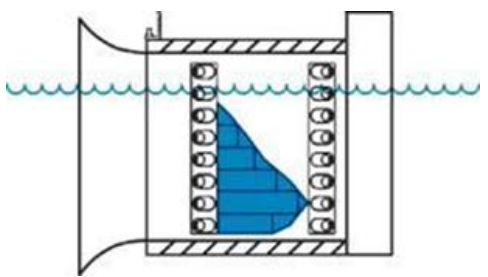

Figura 2. Sistema de medida del caudal

También se han ensayado unas compuertas automáticas para toma en parcela, modelo Baydrive, marca Rubicon Water (Fig. 4). Cada compuerta se compone de una plancha que es movida por un motor, la estanqueidad se consigue mediante una lámina plástica flexible ajustada entre la compuerta y el marco. Se evaluó un tablar con maíz de $187 \mathrm{~m}$ de longitud y $10 \mathrm{~m}$ de anchura, con una pendiente de $0,0032 \mathrm{~m} / \mathrm{m}$ y sin escorrentía libre. Se colocaron dos compuertas en la acequia de la comunidad, una para detener el agua de la acequia y otra para derivar el agua a la parcela. Además se instalaron dos compuertas más en sendos tablares (Fig. 3). Dichas compuertas tienen la función de abrir o cerrar el paso de agua. Se accionaban mediante una aplicación de móvil a distancia, quedando un registro de las 
operaciones realizadas en cada momento. La automatización ha sido parcial ya que por problemas de suministro no se pudo disponer de un sensor de llegada de agua con lo que la apertura y el cierre de las compuertas lo realizaba el agricultor mediante un accionamiento manual. Por consiguiente los resultados que se presentan corresponden a un sistema mecanizado pero activado manualmente.

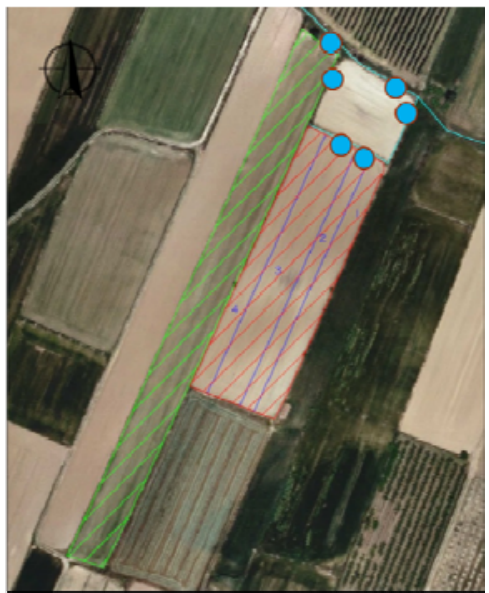

Figura 3. Disposición de las compuertas

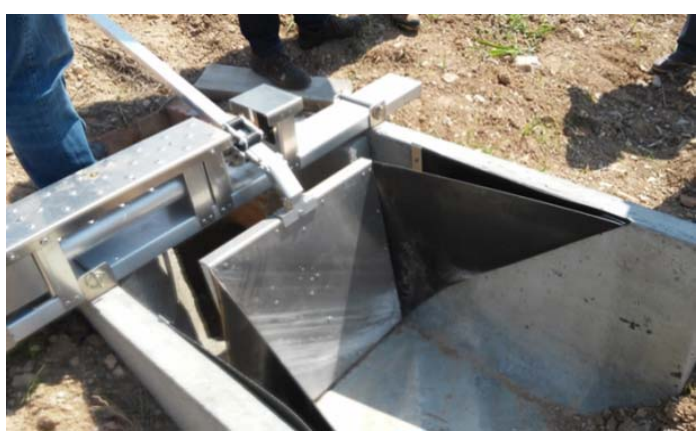

Figura 4. Compuerta Baydrive en parcela

Se evaluaron los riegos siguiendo la metodología y utilizando el aplicativo OPTYM (Monserrat, 1994) que permite determinar la función de infiltración a partir de la medida del avance del agua, la pendiente del tablar, el caudal de entrada y la altura de agua al inicio del tablar. Finalmente para contrastar la función de infiltración obtenida y determinar el perfil de agua infiltrada se utilizó el programa WinSRFR 4.1.3 (Bautista, 2012)

\section{Resultados}

En la figura 5 se muestra la regulación de la compuerta Slipmeter en un ciclo diario, desde su apertura por la mañana hasta el cierre por la noche. La compuerta ha mantenido un caudal muy estable a la salida del embalse. En la apertura se ve un pico pero es corregido rápidamente. Su funcionamiento ha sido correcto durante toda la campaña. Ha supuesto un ahorro considerable de trabajo y viajes al vigilante.

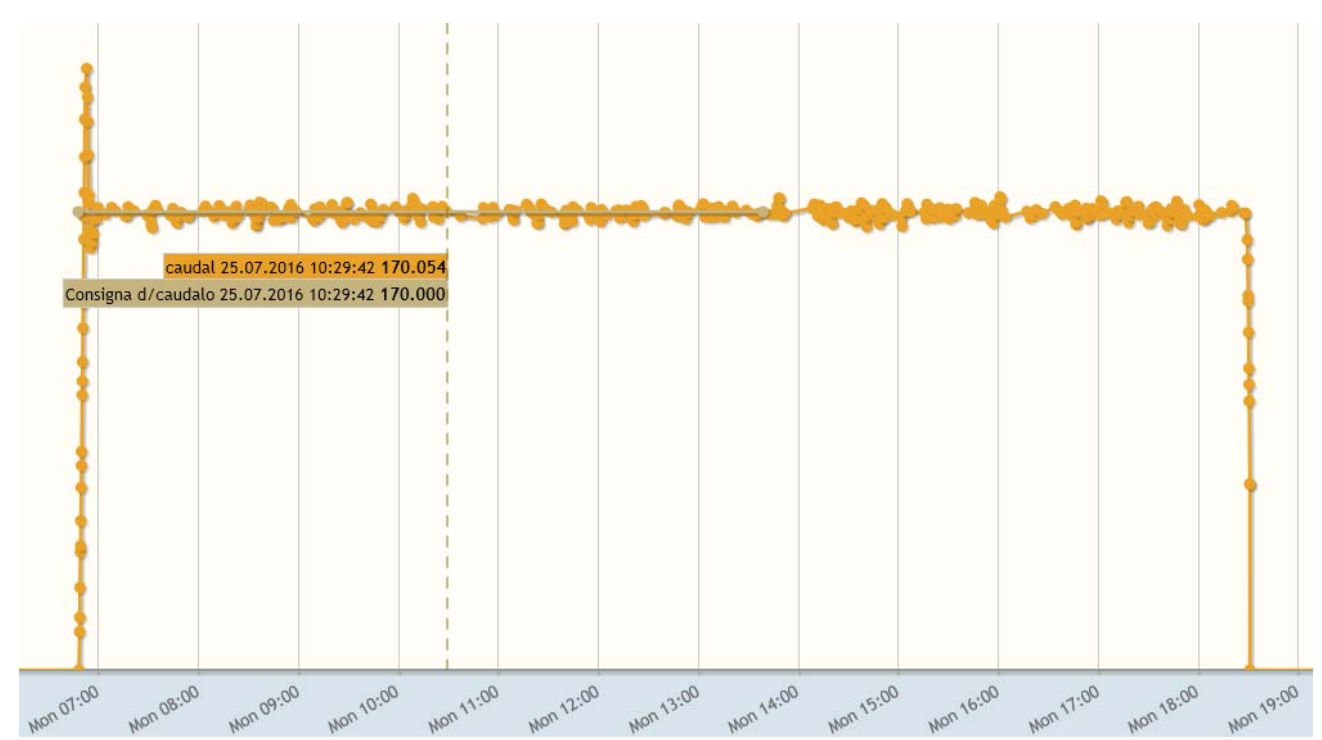

Figura 5. Caudal a la salida de la compuerta Slipmeter durante el ciclo de un día 
En la Figura 6 se representa la dosis media aplicada en los riegos evaluados. Se comprueba que hay una cierta variabilidad en la dosis aplicada con una tendencia a disminuir en los últimos riegos, debido a que se le dijo al agricultor que disminuyera el tiempo de riego.

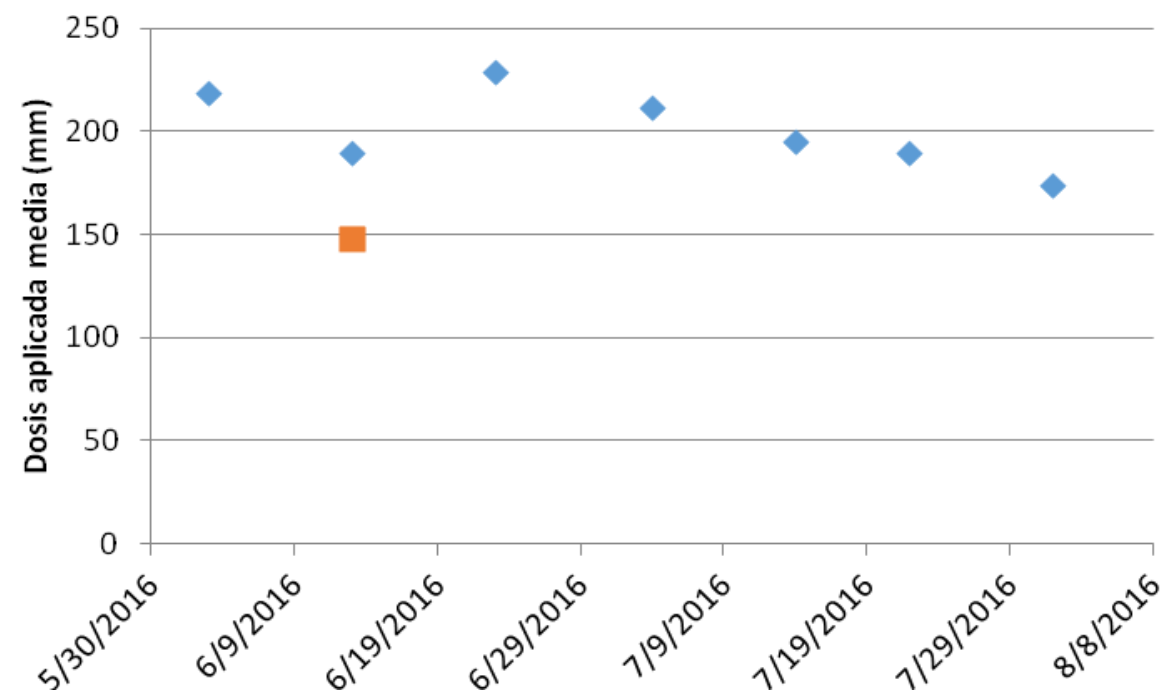

Figura 6. Dosis media aplicada

La función de infiltración obtenida con el programa OPTYM, se contrastó simulando el riego con el programa WinSRFR obteniéndose un buen ajuste del avance y el receso. El programa WinSRFR también proporciona la distribución de agua infiltrada en el tablar (Fig. 7).

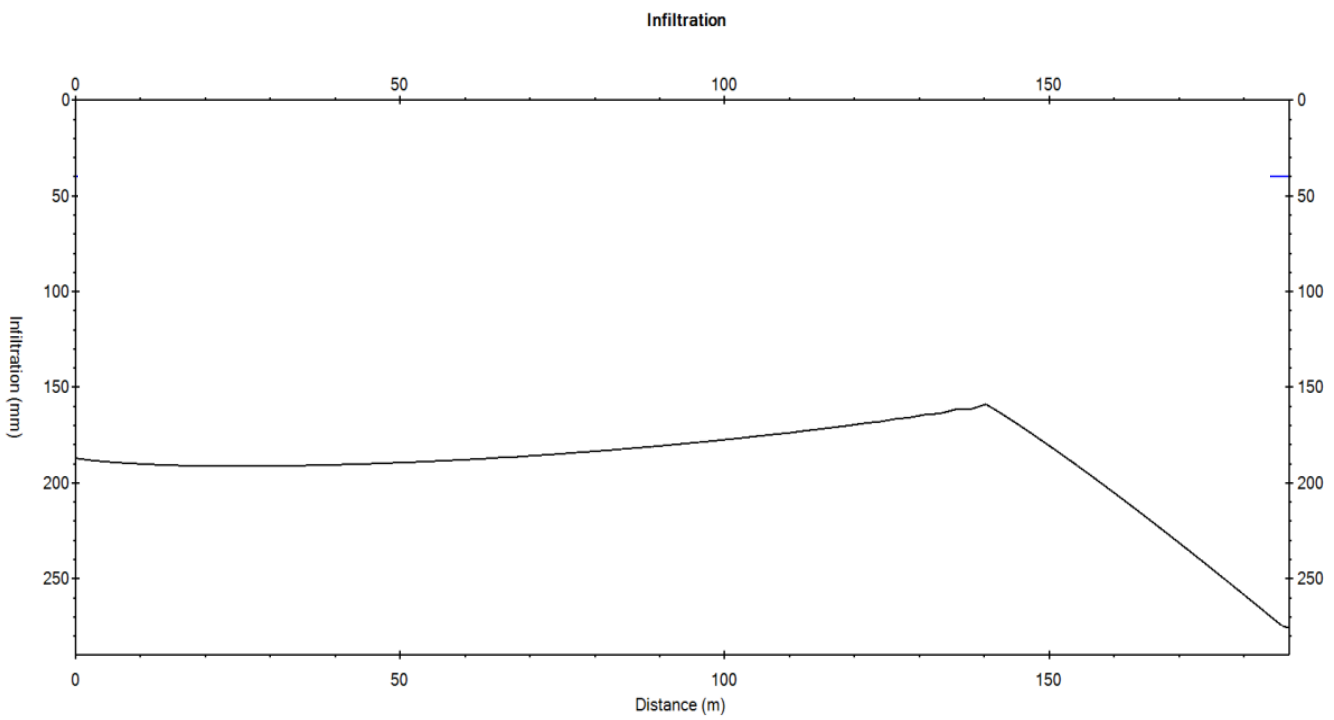

Figura 7. Distribución del agua infiltrada calculada con WinSRFR del riego de 13/6/16

Se comprueba que hay una acumulación de agua al final de la parcela, consecuencia de aplicar agua en exceso. Mediante el programa WinSRFR se calculó el tiempo de riego óptimo para evitar la acumulación de agua en el final, lo que supuso una disminución del 22 $\%$ del tiempo de corte, la dosis resultante se representa en la figura 6 con un cuadrado. Por este motivo se recomendó al agricultor en los últimos riegos que redujera la dosis. 
Los resultados muestran que hay tecnología disponible para la automatización del riego y su manejo óptimo.

El factor final para hacer que la tecnología sea viable es el económico. Para ello se ha desarrollado un procedimiento simple para determinar cuál es el precio máximo de la automatización a partir del cual no es competitiva.

Suponiendo que el único beneficio de la automatización del riego es la disminución de la mano de obra. Si conocemos el coste unitario de contratar la operación del riego durante una campaña $\left(C_{u r}\right)$, que en el caso de los Canales de Urgell es de 138 euros ha- ${ }^{-1}$.

Supongamos que el coste del sistema de automatización, su instalación y mantenimiento es de PM euros, multiplicando este precio por un factor de amortización $(F)$ y dividiendo por la superficie de la unidad de riego $(A)$ tendremos el precio unitario anual que nos supone el sistema. Pues para que esta sea rentable el Precio total del sistema $<P M$. Calculándose $P M$ a partir de la siguiente ecuación,

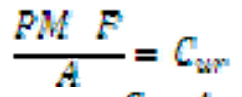

$P M=\frac{C_{w r} A}{F}$

Donde,

$F=\frac{(1+r)^{r} \times r}{(1+r)^{7}-1}$

$r$ : es el precio del dinero (tanto por uno), $T$ : Vida útil de la compuerta (años).

Suponiendo $r=0,05, C u=138$ euros ha ${ }^{-1}$, se ha calculado el $P M$ para diversos tamaños del tablar de riego y vida útil de la instalación.

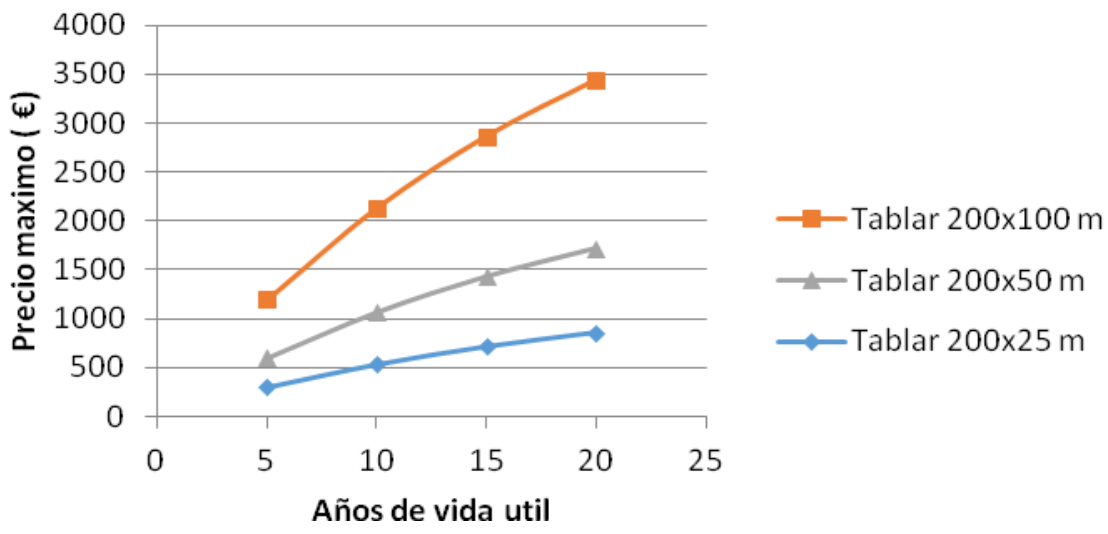

Figura 8. Precio máximo del sistema de automatización para $r=0,05$ y un coste de oportunidad de 138 eur ha $^{-1}$

La conclusión que se puede extraer de la gráfica es que el precio máximo crece a medida que la unidad de riego es mayor, lo cual supone trabajar con mayores caudales si queremos mantener la calidad del riego. Además se observa que los precios del sistema han de ser bastante ajustados para ser competitivos. 
Este precio máximo podría ser mayor si existieran otros beneficios asociados a la automatización como pueden ser el incremento de producción o un ahorro de agua.

\section{Conclusiones}

La compuerta de salida de balsa ensayada ha funcionado correctamente toda la campaña, manteniendo el caudal de salida constante y ahorrado muchos viajes al vigilante.

Las compuertas de parcela ensayadas también han funcionado correctamente, no obstante han sido activadas manualmente debido a que no se ha dispuesto de un sensor de llegada del agua.

Las evaluaciones del riego realizadas muestran que hay una tendencia a aplicar más agua de la necesaria y también hay una variabilidad importante de la dosis media aplicada entre riegos. Estos resultados pensamos que se podrían mejorar si se dispusiera de un sensor de llegada del agua, lo cual podría suponer un ahorro del 10 al $20 \%$ del agua aplicada. Si se consiguiera este ahorro en el resto de parcelas de la comunidad, se podría reducir el turno de riego y esto tendría una repercusión positiva en la producción.

La expresión propuesta para calcular el precio máximo del sistema de automatización es sensible al tamaño del tablar y a los años de vida útil del sistema.

\section{Agradecimientos}

A la empresa Rubicon Water por ceder el material para los ensayos, a la Comunidad General de regantes de los canales de Urgell, al Departamento de Agricultura Ramaderia, Pesca i Alimentació de la Generalitat y al sindicato de riegos de Arbeca por su colaboración en la ejecución y seguimiento del proyecto.

\section{Bibliografia}

Bautista, E., Schlegel, J.L., and Strelkoff, T.S. (2012). WinSRFR 4.1 - User Manual. USDAARS Arid Land Agricultural Research Center. 21881 N. Cardon Lane, Maricopa, AZ, USA.

Cots, L. , Clop, M., Lopez, R., Sangrà J, Esteban M, Barragán JD, Cuadrat O (2007). Indicadores de la gestión del agua en los canales de Urgell. Riegos Y Drenajes XXI, (156), 26-32. Retrieved from http://repositori.udl.cat/bitstream/handle/10459.1/46526/011921.pdf?sequence=1\&isAllo wed=y

Monserrat, J. (1994). Solución al problema inverso del riego por tablares mediante un modelo hidrológico mixto. Tesis doctoral. Universidad de Lleida. Retrieved from www.cervantesvirtual.com/.../solucion-al-problema-inverso-del-riego-por-tablares

Monserrat, J., Rosell, J., Barragán, J., Cots, L., \& Pastor, R. (1998). Sistema semiautomático para riego por superficie. Riegos Y Drenajes $X X I,(102), 39-41$. Retrieved from http://repositori.udl.cat/bitstream/handle/10459.1/47411/006948.pdf?sequence=1 